\title{
Local search to improve coordinate-based task mapping
}

\author{
Evan Balzuweit ${ }^{\mathrm{a}}$, David P. Bunde ${ }^{\mathrm{b}}$, Vitus J. Leung ${ }^{\mathrm{c}}$, Austin Finley ${ }^{\mathrm{b}}$, Alan C.S. Lee ${ }^{\mathrm{b}}$ \\ ${ }^{a}$ Department of Computer Science $\mathcal{E}$ Engineering \\ Washington University in St. Louis, St Louis, MO, USA \\ ${ }^{b}$ Department of Computer Science \\ Knox College, Galesburg, IL, USA \\ ${ }^{c}$ Sandia National Laboratories \\ Albuquerque, NM, USA
}

\begin{abstract}
We present a local search strategy to improve the coordinate-based mapping of a parallel job's tasks to the MPI ranks of its parallel allocation in order to reduce network congestion and the job's communication time. The goal is to reduce the number of network hops between communicating pairs of ranks. Our target is applications with a nearest-neighbor stencil communication pattern running on mesh systems with non-contiguous processor allocation, such as Cray XE and XK Systems. Using the miniGhost mini-app, which models the shock physics application CTH, we demonstrate that our strategy reduces application running time while also reducing the runtime variability. We further show that mapping quality can vary based on the selected allocation algorithm, even between allocation algorithms of similar apparent quality.
\end{abstract}

Keywords: Task mapping, stencil communication pattern, non-contiguous allocation, local search.

\section{Introduction}

The resource management pipeline for parallel batch jobs has three stages, scheduling, allocation, and task mapping. Scheduling decides when a job will run, allocation determines which nodes it is assigned, and task mapping matches the job's tasks to individual computational elements (nodes or cores). Scheduling and allocation are typically done at the system level, while task mapping is left to the application. In MPI programs, task mapping is the decision of which MPI rank performs each part of the computation, even if done in a "default" manner.

This paper focuses on improving task mapping, which has been shown to significantly reduce job running times for a variety of scientific applications (e.g. [1, 2, 3, 4, 5]), including achieving a speedup of 1.64 on a quantum system simulation [3]. Better task mapping reduces the number of hops between communicating tasks and hence the amount of system bandwidth consumed by each message. As systems grow larger and processor performance continues to improve faster than network performance, its importance will continue to grow.

In addition to performance, we consider the effect of task mapping on run-time predictability, which has recently become the subject of active research (e.g. [6]). Predictability is desirable in HPC systems because users must estimate job running time for the scheduler.

The job's communication pattern and the system's network topology are both important for task mapping. Here, we focus on jobs communicating in a nearest-neighbor stencil pattern, a 
very common pattern in computational science applications. In this pattern, the tasks correspond to integer points in a grid and communicate with their nearest neighbors, the 4 closest points in the $+x,-x,+y$, and $-y$ directions for $2 \mathrm{D}$ or the 6 closest points for $3 \mathrm{D}$ (add the $+z$ and $-z$ directions). This pattern arises naturally from spatial decompositions into rectangular ${ }^{1}$ regions.

In this paper, we target machines whose network topology is a 3D mesh and allow the possibility that a job is allocated to a non-contiguous set of nodes. This is appropriate for the Cray XT, XE, and XK series of systems, including the Cray XE6 (Cielo) we use for our experiments. Two kinds of allocators, linear and center-based, have been studied for this type of machine. We study the interaction of our task mapping algorithms with both types of allocators using experiments (Cray uses a linear allocator) and simulations.

Although jobs are allocated nodes on our target machines, our task mapping algorithms work in terms of MPI ranks rather than compute nodes allocated to that job. Each MPI rank is a single process in a distributed memory program; we will refer to them simply as ranks. In general, each compute node may support many ranks depending on its number of cores and the mix of distributed- and shared-memory programming models (e.g. MPI and OpenMP) used.

A recent task mapping algorithm developed for our setting is Geometric (Geом) [7], which operates by finding corresponding decompositions of the job tasks and allocated ranks. This algorithm was shown to outperform a wide variety of other algorithms, reducing application running time by around $30 \%[7,8]$.

Contribution. The main contributions of this paper are as follows:

- We present a new algorithm, Geom-Based Local Search (GSearch), that tries to improve on Geom using the observation (e.g. [7, 9, 10, 8]) that job running time correlates with the average number of hops between communicating tasks (average hops metric). Specifically, GSEARCH uses a local search to improve the average hops metric by swapping pairs of tasks when doing so improves the average hops metric.

- We demonstrate our algorithm in a proxy application and show that it improves total application running time. Furthermore, it does so while reducing the running time variability.

- We show that the benefit of GSEARch depends on the algorithm used to allocate nodes to jobs. While the benefit is fairly modest with linear allocation algorithms, it is much greater with a center-based algorithm. This is the first time such a dependence has been seen.

- We examine the number of swaps made by GSEARCH, showing it is reasonable in practice. We also show that it can be large, but suggest using a cutoff to avoid pathological cases.

At a high level, our results again demonstrate that GЕом is a good task mapping algorithm for practical settings, but show that local search (represented by GSEARCH) can improve upon it.

This paper builds on a preliminary version [11], with the addition of center-based allocation, more analysis of the variability of mapping quality, and improved presentation.

The rest of this paper is organized as follows. Section 2 describes our algorithms. Section 3 describes the setup for our experiments and simulations. Section 4 describes our results. Section 5 summarizes related work. Section 6 concludes and discusses future work.

\footnotetext{
${ }^{1}$ We use "rectangular" to include rectangular prisms in 3D.
} 


\section{Algorithms}

We begin by reviewing allocation algorithms and then showing how GSEARch builds on Geом.

\subsection{Allocation}

To identify the nodes allocated for each job, we use two different allocation algorithms. The first of these is a linear allocation algorithm that combines ideas of Lo et al. [12] and Leung et al. [9]. This algorithm organizes the nodes in a linear order along an "s-curve", which curves back and forth along the machine's shortest dimension $(z)$. The free nodes are grouped into intervals by their position along the curve and the algorithm allocates nodes from the smallest interval with enough nodes (best fit). If no interval is large enough, nodes are chosen to minimize the maximum distance along the curve between chosen nodes. This scheme is fast and generates good allocations [13]. It is also similar to the algorithm used in practice on Cray systems [14].

Our second allocation algorithm is MC1x1 [15], which builds a candidate allocation around each available node. The node being built around is the center and its candidate allocation is the closest nodes to the center (measured in hops). The candidate allocation minimizing the sum of pairwise distances is selected. Because of its selection criteria, in some sense MC1x1 is optimizing all-to-all communication, essentially being pessimistic in the absense of job-specific information. MC1x1 is based on a similar algorithm MC [16] and several variations have been studied in the literature (e.g. [17, 18]). It is not used in practice, but represents an approach which avoids the 1-dimensional reduction used by the linear allocation algorithms.

\subsection{Task mapping}

We now describe our task mapping algorithms. Geом first rotates the job so that its dimension lengths have the same order as the bounding box of the ranks (i.e. if the bounding box of ranks is largest in the $x$ dimension, then the job's largest dimension will also be $x$ and so on).

After any necessary rotations, Geom develops corresponding decompositions of the set of tasks in the job and the set of ranks allocated to it. Each set is represented with a list of coordinates, the coordinates of a task being its position in the job's communication pattern and the coordinates of a rank being its position in the machine's 3D grid. In addition, the job is represented with a triple giving its size in each dimension; the job is always a rectangle. At a given step, the job's tasks are split into two rectangles as evenly as possible along its longest dimension. For example, a $3 \times 4 \times 5$ job would be split into rectangles of size $3 \times 4 \times 3$ and $3 \times 4 \times 2$ respectively. The same dimension is then used to split the ranks into two subsets with the corresponding sizes. In the example above, the first subset would have the 36 ranks with smaller $z$ coordinates and the second would have the 24 ranks with larger $z$ coordinates (ties broken consistently). The corresponding subsets of tasks and ranks are then mapped to each other recursively. The base case is subsets of size one, for which the only task is mapped to the only rank.

GSEARch begins with the mapping generated by Geom and examines pairs of tasks, swapping the ranks executing them when doing so reduces the average hops metric. Figure 1 shows pseudocode for the search part of GSearch. Note that GSEarch performs improving swaps as it finds them in the (serial) nested loops; more sophisticated strategies were considered and discarded as described in Section 4.4. We discuss a parallel implementation in Section 6.

For a fast implementation, the test of whether a swap reduces the average hops (line $\star$ in Figure 1) should not calculate the overall average hops metric, but just compare the distances involving the tasks that were swapped since only these changed. This optimization is used in 


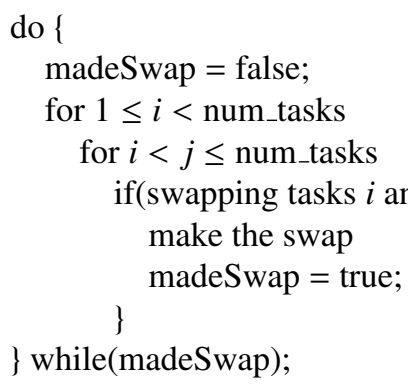

Figure 1: Pseudocode for local search component of GSearch. This version does not limit the number of swaps made and performs improving swaps as it finds them; see Sections 4.3 and 4.4 for discussion of more sophisticated strategies. The line marked with $\star$ admits multiple implmentations, discussed in this section (2.2).

our implementation since it reduces the time to compute the effect of a swap from quadratic (examining all pairs) to constant.

In GSEARCH as shown in Figure 1, the search continues until a local minimum is found, at which point no pairs can be swapped to improve the metric. We discuss setting a limit on the number of swaps made in Section 4.3.

\section{Experimental setup}

We evaluate these algorithms with experiments on a large system and on a trace-based simulator. The simulations did not model the effect of mapping on running time so we evaluate the algorithms with average hops. The simulations were as comparable to the experiments as possible, with a similar machine size and interconnect topology. The simulations let us run more jobs, more closely instrument the jobs, and try center-based allocation (not used on our test machine).

\subsection{Cielo}

Our experiments were run on the ACES [19] system Cielo [20], a Cray XE6 with 8,944 compute nodes plus a number of service nodes connected in a Cray Gemini 3D torus in a sixteen by twelve by twenty-four (XYZ) topology, with two nodes (sockets) per Gemini. Each compute node is a dual AMD Opteron 6136 eight-core "Magny-Cours" socket G34 running at $2.4 \mathrm{GHz}$. Each service node is a 272 AMD Opteron 2427 six-core "Istanbul" socket F running at $2.2 \mathrm{GHz}$. The bi-section bandwidth is 6.57 by 4.38 by 4.38 (XYZ) TB/s. As of June 2014, Cielo was number 32 on the Top500 list [21].

The application used in the experiments was miniGhost [22]. As part of the exascale research program, the DOE lab community is developing mini applications (miniApps) to represent the computational core of major advanced simulation and computing codes. MiniGhost is a miniApp for exploring boundary exchange strategies in stencil computations. The miniGhost application is a bulk-synchronous message passing code whose structure is modeled on the computational core of CTH [23], a multi-material, large deformation, strong shock wave, solid mechanics code.

A set of experiments consists of miniGhost runs for various numbers of nodes (powers of 2). In each experiment, the mapping algorithms are run one after the other with the same allocation to minimize the experimental variances other than the mapping algorithm used. All experiments in a set were submitted to the system queue at roughly the same time. We ran six sets. 


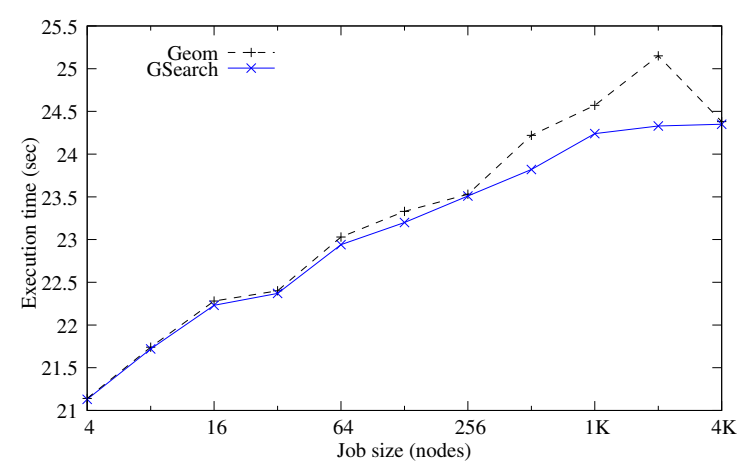

Figure 2: Running time by job size for miniGhost on Cielo. (Average over 6 sets of experiments)

The communication pattern for each job was a 3D nearest neighbor stencil. A job with $2^{k}$ tasks ran with dimensions $2^{k / 3} \times 2^{1+(k+1) / 3} \times 2^{(k-1) / 3}$. These dimensions were used for this application in prior work on task mapping [7, 24]; the aspect ratio comes from a shaped charge problem for $\mathrm{CTH}$. We ran jobs with sizes ranging from 4 to $4 \mathrm{~K}$ tasks. Internode communication was performed with MPI. Each MPI rank ran on all 16 cores in a node, with intranode parallelism managed with OpenMP. The application spends about thirty percent of its time communicating.

\subsection{Simulator}

For our simulation, we ran a trace-based simulator [25] with the LLNL-Atlas trace (version 1.1 clean) from the Parallel Workloads Archive [26]. The trace is from a cluster with 9,216 nodes. To match this node count with a mesh system, our simulation treated it as a $24 \times 24 \times 16$ mesh. From this trace, we took job start times, execution times, and number of nodes needed. (Note that most Parallel Workloads Archive traces give job arrival times and thus require scheduling; since the LLNL-Atlas trace gives start times, every job runs exactly when it did on the real system.)

The Parallel Workloads Archive traces do not record job communication patterns. Since this paper focuses on nearest-neighbor stencil patterns, we assumed that every job used this pattern. For a specific job size, we considered all rectangular shapes with the correct number of processors and selected the one with the smallest bounding cube. In a sense, this is the "roundest" possible job shape; we used it since the simulated machine's dimensions also have similar lengths.

\section{Results}

Now we present our experimental results in four parts. First, we demonstrate that GSEARCH yields better task mappings than GEOM. Second, we show that it also gives more predictable performance. Third, we look at the number of swaps that GSEARCH makes, a potential concern for its running time. Fourth, we look at a couple of slight variations on GSEARCH that were designed to reduce the number of swaps needed.

\subsection{Average mapping quality}

The main criteria for task mapping quality is application running time. Figure 2 shows the average running times for miniGhost runs of different sizes using GeOM and GSEARCH. The two algorithms are essentially tied at small job sizes, with GSEARCH gradually becoming better as the 


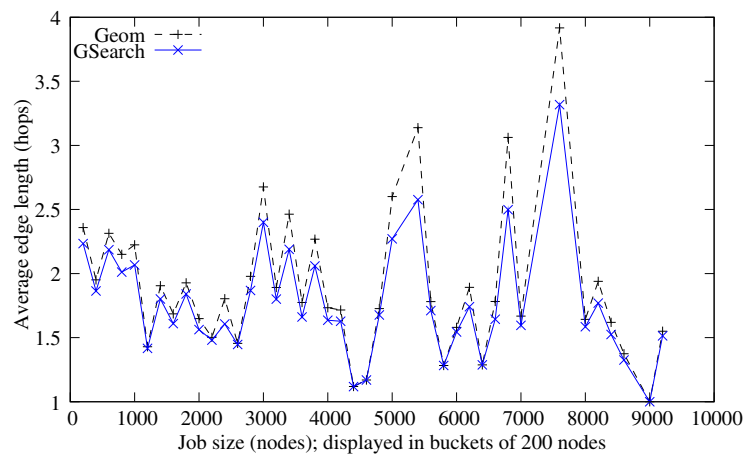

Figure 3: Average edge length by job size for LLNL-Atlas trace with linear allocation

job size increases to $2 \mathrm{~K}$ nodes and then essentially tying again at $4 \mathrm{~K}$ nodes. GSEarch gives a better average time for all these sizes, with the largest difference being 0.83 seconds at $2 \mathrm{~K}$.

The relatively close performance of the two algorithms at $4 \mathrm{~K}$ nodes breaks the apparent performance trends up to that point. In particular, it appears anomalous since the running time of Gеом breaks its clear upward path prior to that job size. One possible cause of this variation is random noise due to contention from other jobs, which is known to significantly affect running times [6]. An examination of individual runs does reveal significant variation, but not in a way that supports this explanation, however. In only one of the six runs at $4 \mathrm{~K}$ nodes did Geom take longer than 24.25 seconds, its average time at $2 \mathrm{~K}$ nodes. Even the best $4 \mathrm{~K}$ run for GSEarch relative to Geom only beat it by 0.52 seconds, less than the average amount by which GSEARCH beat Geом for the $2 \mathrm{~K}$ node size. The apparent anomaly of Geom's performance at $4 \mathrm{~K}$ is an interesting open issue and we return to it in Section 6.

Our simulation results also support the idea that GSEarch improves upon Geom task mappings. Figures 3 and 4 compare the algorithms using the average distance metric as a function of job size for the two allocation algorithms; recall that the simulations do not model the effect of mapping on running time but that average hops has been shown to correlate with it. Since GSEARCH explicitly optimizes this metric, it always achieves average hops that are at least as low as Geom. Figures 3 and 4 do not show the steadily increasing improvement depicted in Figure 2 , but we believe the specific peaks and valleys are artifacts of the allocations received by jobs; note that the different allocation algorithms generated peaks for different jobs sizes. For both allocation algorithms, the curves for Geom and GSEarch are roughly parallel, suggesting that variations are caused by allocation quality, which affects both task mapping algorithms.

Figures 3 and 4 do reveal significantly different behavior for the two allocation algorithms. GSEARCH provides much greater improvements in average mapping quality on allocations generated by $\mathrm{MC} 1 \mathrm{x} 1$ than ones generated by the linear algorithm. In addition, both mapping algorithms deliver worse mappings on the allocations generated by MC1x1. Since Figure 3 also shows GSEARch tending to give larger improvements when Geom's average hop count is higher, these observations may be related; the worse a mapping that Geом finds, the more potential improvement available to GSEARCH.

A closer look at the data reveals that although GSEARCH gives greater average improvements for MC1x1-generated allocations, it does this while improving fewer jobs. GSEARCH's mapping is strictly better than Geom's on $49.7 \%$ of the jobs for linear allocation, but only $25.6 \%$ of them for 


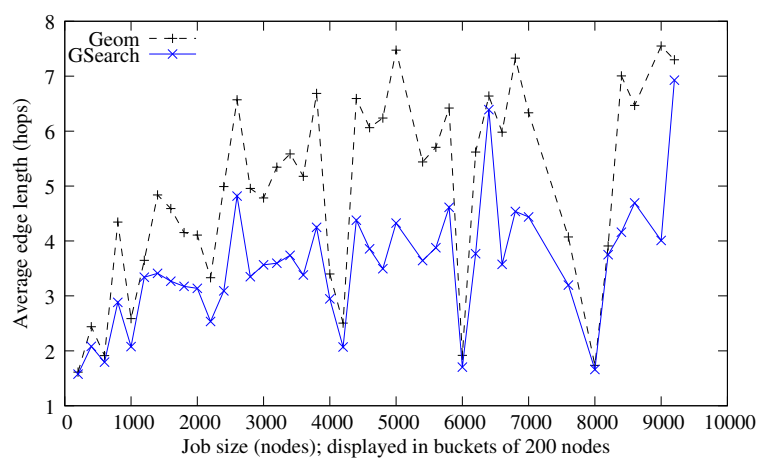

Figure 4: Average edge length by job size for LLNL-Atlas trace with MC1x1 allocation

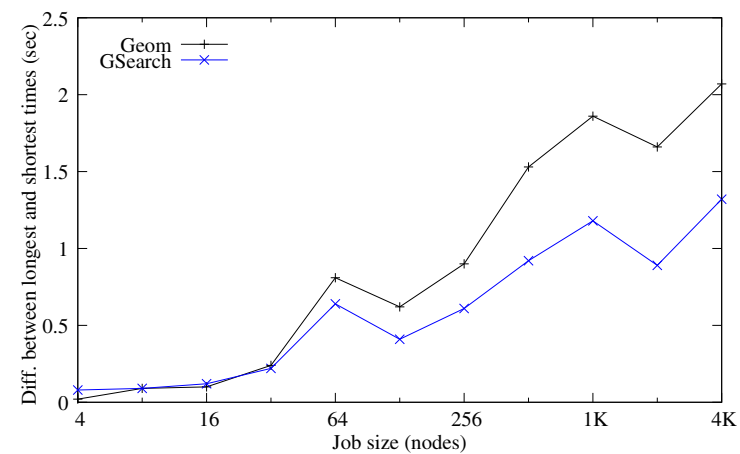

Figure 5: Difference between max and min running time by job size for miniGhost on Cielo

MC1x1 allocation. Thus, MC1x1's allocations are more likely to lead GEom to a local optimum, but its failures are much worse, leading to an overall worse performance. This has a similar flavor to a phenomenon previously found for allocation: optimizing individual jobs to minimize the all-pairs pairwise distance between allocated nodes led to worse results on this same metric for a stream of jobs [15].

Despite the differences between our experiments and each of the two sets of simulations, all three either directly or indirectly indicate that GSEARCH improves job average running time.

\subsection{Variability}

A second criteria for task mapping quality is predictability of performance. Figure 5 shows the difference between the longest and shortest running times for each algorithm on each job size for the Cielo experiments. We see that the range of running times increases with job size for both algorithms, but that GSEARCH generally delivers only about two thirds of the runtime variability of Geom. Thus, the performance of GSEARCH seems to be both slightly better and more predictable.

We saw some decrease in variability in the simulations as well. Figures 6 and 7 show the difference between the best and worst average edge lengths for jobs of various sizes in the simulations. Again, GSeArch generally delivers lower values. Unlike in the experiments, however, the variability decreases as jobs grow. We attribute this to the metric, which averages differences 


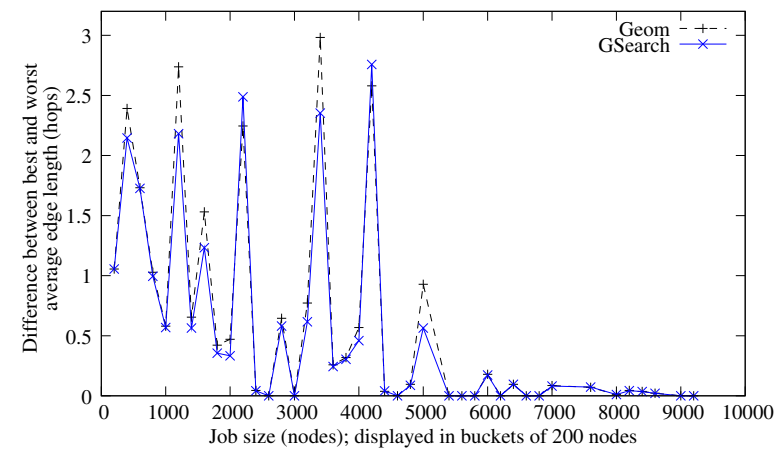

Figure 6: Difference between best and worst average edge length by job size for LLNL-Atlas trace with linear allocation. Each point is greatest difference for sizes in a 200 -node range

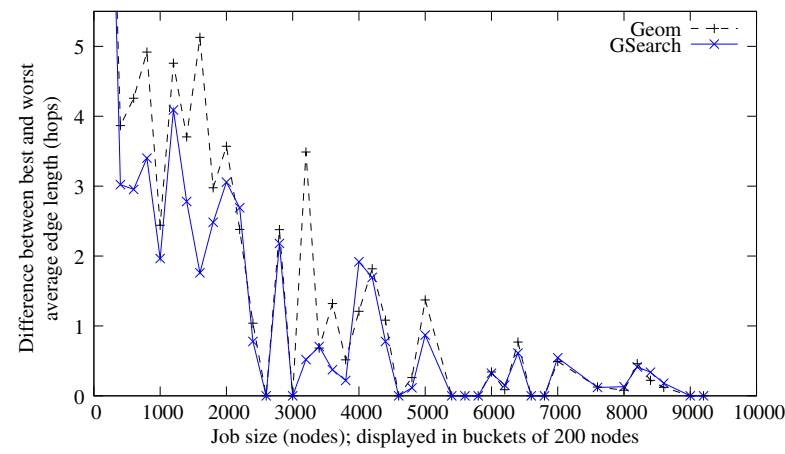

Figure 7: Difference between best and worst average edge length by job size for LLNL-Atlas trace with MC1x1 allocation. Each point is greatest difference for sizes in a 200-node range. Omitted point at size 200 has difference 10.57

over more edges as the jobs grow. The figures also suggest a difference in the variability based on the allocator as well, though it is possible that the greater improvement in variability seen with MC1x1 allocation occurs because of the increased average edge length with that allocator.

Other support for GSEARCH providing less variability than GEOM is also provided in the average edge length results presented in Figures 3 and 4. Much "random" variability in job running times is caused by congestion on network links, which can delay messages [6]. Reducing the number of hops between a pair of communicating tasks makes that pair's messages less susceptible to congestion. It also makes that pair's messages less likely to interfere with other messages in the system, including messages between other tasks in the same job.

\subsection{Number of swaps needed}

Having discussed mapping quality, we now examine the number of swaps made by GSEARCH. This was one of our initial concerns about GSEARCH; if the number of swaps is too large, the extra time spent improving the task mapping would eliminate the benefit of doing so. In order to study this issue, we looked at the numbers of swaps used in our experiments and simulations, as well as performing additional simulations specifically to get a sense of the distribution of swap frequencies. This work assuaged our concerns and suggested bounds that could be used to limit the number of swaps and avoid extreme worst-case behavior. 


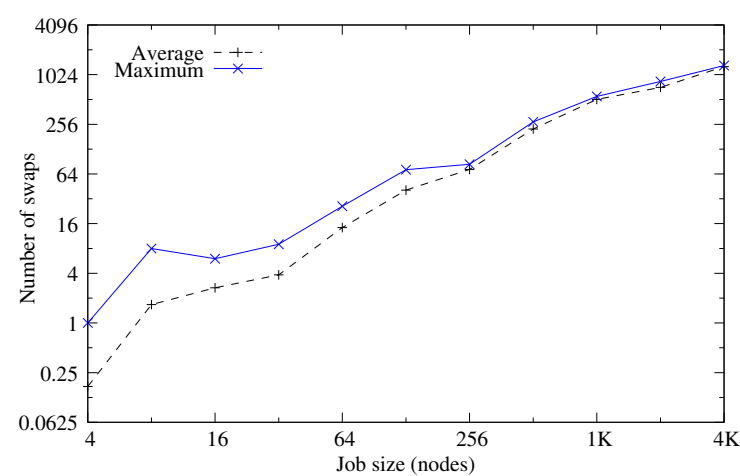

Figure 8: Number of swaps made by GSEARCH as a function of job size (average and max over 6 sets of Cielo experiments)

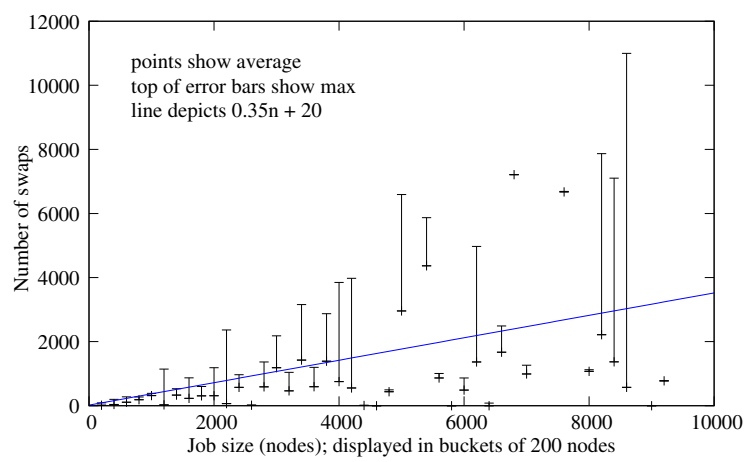

Figure 9: Number of swaps made by GSEARCH as a function of job size on LLNL-Atlas trace using linear allocation

Figure 8 shows the average and maximum number of swaps GSEARCH performed on jobs of each size during our experiments on Cielo. Both the average and maximum values seem to grow linearly with the job size. The difference between them shrinks in proportion of the average value, though its absolute magnitude generally increases with job size (not visible from the graph because of the log-log scale).

The simulations using linear allocation show very similar trends, albeit with considerably more noise. Figure 9 shows the number of swaps as a function of job size; the points give the average number of swaps and the top of the error bar shows the maximum value seen in our simulation. Again, the average number of swaps seems to increase linearly with the job size, with most points below the line of slope 0.35 . The main exceptions are points representing few jobs. The upper envelope of the maximum numbers of swaps also seems to be growing linearly.

The simulations with MC1x1 allocation tell a different version of the story; see Figure 10. Again, the average and maximum numbers of swaps seem to increase linearly if we ignore occassional noise. Now, however, the slope is much greater; the line depicts slope 3 . The greater number of swaps needed may reflect the fact that GSEARCH provides a greater improvement, but that does not solve the mystery of why the allocation algorithms behave so differently.

In addition to the trace-based simulations, we ran a couple of other types of simulations. First, we looked at the number of swaps made by GSEARCH on a randomly-chosen allocation of nodes. 


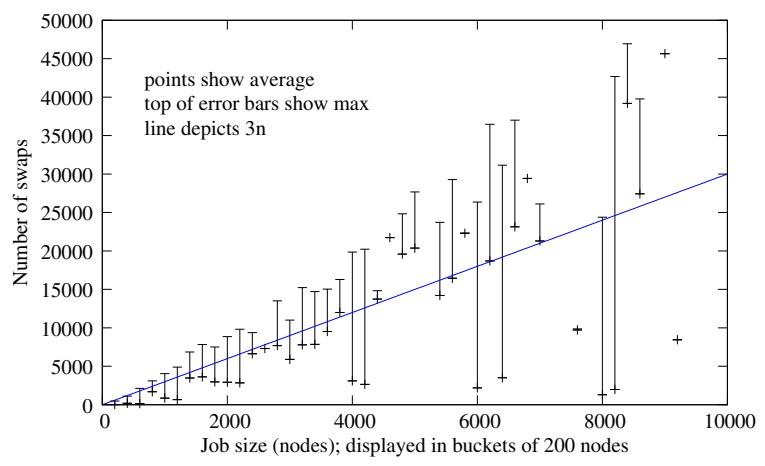

Figure 10: Number of swaps made by GSEARCH as a function of job size on LLNL-Atlas trace using MC1x1 allocation

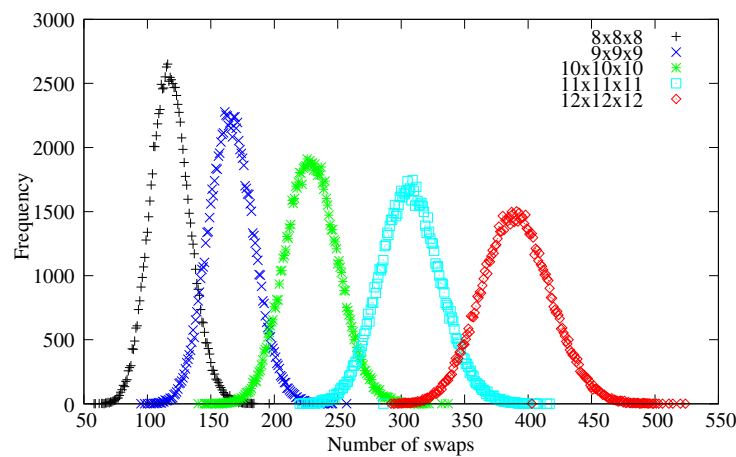

Figure 11: Swap count frequencies from 100,000 random allocations on $16 \times 24 \times 24$ system

We tried a variety of job sizes, running each for 100,000 allocations. Figure 11 plots the number of allocations that resulted in each number of swaps. All sizes give bell-shaped frequency curves, indicating that the number of swaps is fairly concentrated about the mean value. As the job size increases, the curves move to the right and do flatten somewhat, but the worst we saw for any of these curves was one allocation 133 swaps above the mean (for the $12 \times 12 \times 12$ curve). The mean number of swaps was consistently a bit below $n / 4$ for a job of size $n$ and all observed values used at most $0.35 n+20$; this is the line plotted in Figure 9. Thus, the random allocations behave much more like the linear allocations than the ones generated by MC1x1.

Knowing that random searches do not easily find unlikely worst case situations, we specifically tried to find small cases where GSEARCH used a large number of swaps. We exhaustively ran GSEARCH on all allocations of a $4 \times 2 \times 1$ job on a $4 \times 4 \times 2$ machine. It can require as many as $12(=1.5 n)$ swaps, but only in 4 of $\left(\begin{array}{c}32 \\ 8\end{array}\right)=10,518,300$ possible allocations. We also hand constructed some bad mappings and found improving sequences of swaps with length up to $2.58 \overline{5} n$ ( 31 swaps for a $3 \times 4$ job). This is closer to the behavior observed with MC1x 1 , but our failure to reproduce the large numbers of swaps suggests that it only happens with larger jobs.

We also sought an absolute bound on the number of swaps that are possible. One idea is to bound the number of times a given pair of tasks can swap. Surprisingly, we observed several cases in which a pair of tasks can swap more than once, with the second swap putting the tasks back onto their original ranks. This can happen when intervening swaps move their neighbors in 


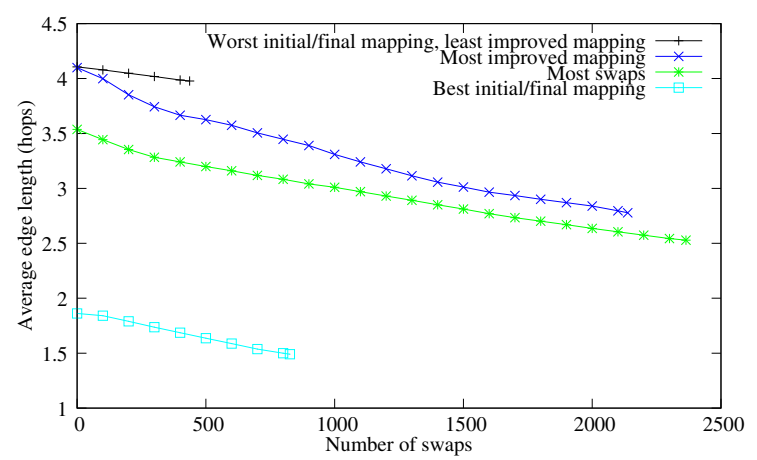

Figure 12: Average edge length as a function of the number of swaps made on sample jobs from the LLNL-Atlas trace with linear allocation. All selected jobs used 2,144 nodes

a way that makes the original swap detrimental.

Since we do not have a firm bound on the number of swaps that GSEARCH will make, a natural idea for implementations is to include a limit on the number of swaps. This raises the question of how the benefit of swaps is distributed; do a fraction of the swaps have disproportionate impact on the results? To investigate this, we had our simulator calculate the average hops metric after each 100 swaps. Figure 12 shows the results for some jobs of size 2,144 using the linear allocator; these jobs were selected because of the variety of behavior they exhibit. Depicted are four jobs (out of 12 of this size) representing extremes in GSEarch's behavior on jobs of this size. The first job received the worst initial mapping from Geom, was least improved (as a \%) by the local search, and had the worst final mapping. The others improved the most, required the most swaps, and received the best initial and final mappings, respectively. The amount of improvement for most jobs was fairly similar in each 100 swaps, but there was some variation. (Having exactly the same improvement would give a straight line in the figure.) Different jobs also improved at significantly different rates (appearing as different slopes) in a way that does not appear to be related to the quality of the initial mapping; the lines for some of the jobs not shown in Figure 12 actually cross. (Recall that the depicted jobs were selected because they are boundary cases.)

Despite the variety of behavior appearing in Figure 12, the benefit from GSearch's search strategy seems to be roughly proportional to the number of swaps. We attempted to reproduce Figure 12 on Cielo with a series of runs with different limits, but random noise dominated the results despite jobs in an experiment running consecutively with the same allocation. Even so, we conclude that using a limit does not appear to harm GSEARCH's performance disproportionately, which is the point since the goal of a limit is to prevent pathological behavior.

\subsection{Variations on GSEARCH}

Thus far, this paper has discussed a particular local search strategy which makes any swap that improves the average distance as soon as it is identified. Early in this research, we used simulations to compare this algorithm (GSEARCH) with a couple of alternate ways to choose swaps. In particular, we looked at the following alternatives:

1. Consider swaps rank by rank, testing all swaps involving a given rank before making the best one (i.e. the one which improves the average distance by the most).

2. Test swaps of all pairs before making the single best one. 
The goal of each of these alternatives was to reduce the number of swaps by avoiding long sequences of swaps that provide little benefit. We found that the alternate algorithms accomplish this, reducing the number of swaps performed by $12 \%$ and $35 \%$ respectively while giving final mappings that are of essentially indistinguishable quality (average distance within $0.2 \%$ ). Unfortunately, the additional time required to find each of the swaps eliminated the savings of doing fewer of them; the first alternative took 2\% longer than GSEARCH and the second took 2.5 times as long. The simple strategy used by GSEARCH seems to be the best of these ideas.

\section{Related work}

An alternate allocation model is used by systems such as the Blue Gene, which always assigns each job a contiguous set of nodes isolated from each other [27]. This isolation provides benefits [6], but also decreases system utilization (e.g. [12, 28]). There are task mapping algorithms designed specifically for systems with contiguous allocation (e.g. [29, 10]). Note that our algorithms can be applied to the contiguous setting as well, but that the reverse is not true.

There is a wide variety of prior research on task mapping in different settings. Before Geom, the main algorithms for task mapping with non-contiguous allocations were based on linear orderings. MiniGhost's default behavior assigns tasks to ranks in row-major order; this is typical for applications that do not specifically consider task mapping. With ALPS and Moab, MPI ranks are determined by the allocation order (along the space-filling curve). The default mapping then assigns rows along this curve, which can create long edges in the task columns and at the end of each row. Barrett et al. [24] observed that this mapping did not scale well above 256 nodes on Cielo and improved it by renumbering tasks so that each node is assigned a submesh of the job.

Geom was proposed by Leung et al. [7], who originally called it RCB. They showed that it beat the strategies above and adaptations of task mapping algorithms from the contiguous setting proposed by Bhatelé et al. [10]. Deveci et al. [8] created Multi-JAGGed (MJ), a slight generalization of GEOM that uses multi-way partitions instead of bisections to decompose the sets of tasks and ranks. They also considered shifts to account for the wraparounds of a torus interconnect and trying all rotations rather than just the one that gives the job and ranks the same dimension ordering. The shifts and extra rotations gave a slight benefit to miniGhost and more for miniMD [30], a miniapp based on a molecular dynamics application whose communication pattern can propagate information further in a single time step.

Hoefler and Snir [31] describe a recursive bisection algorithm for general job communication patterns. They represent the communication pattern with a graph and use METIS [32] to split the graph. Often generality is preferable, but in this case GEOM has two important advantages over the more general algorithm. First, it is a simpler and faster algorithm. Second and more importantly, it gives better mappings; Deveci et al. [8] showed that MJ resulted in job communication times roughly $15 \%$ shorter than the general algorithm. We attribute this advantage to the benefit of using task coordinates at every level of the recursion. Consider Figure 13, which shows two levels of a possible Gеом decomposition. Because task coordinates are used, tasks with low $x$ coordinates are near each other (in regions $A$ and $C$ ) despite being separated by the decomposition's first cut. This is not necessarily true if a general graph partitioner is used since reversing the roles of $C$ and $D$ or using different dimensions to split the tasks and ranks could give equally good cuts. Using coordinates allows Gеом to easily exploit the full problem structure.

Others have proposed local search using swaps that improve the current mapping, though only in the context of the unstructured problem where the job communication pattern is an arbitrary graph. Bokhari [33] alternated stages of local search with making random swaps. Lee and 


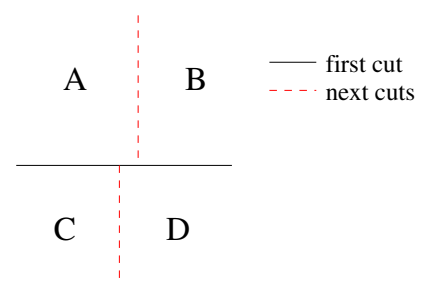

Figure 13: Two levels of cuts in decomposition created by Geom.

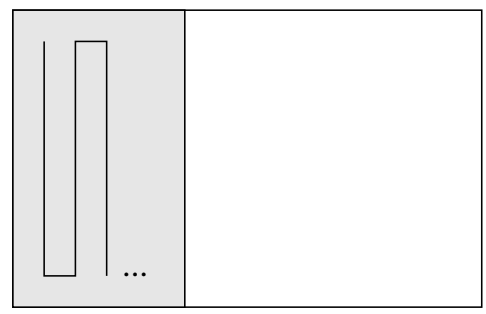

Curve-based allocation

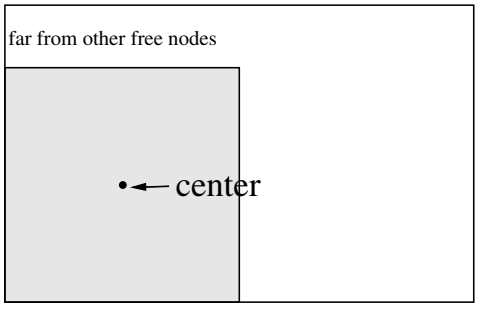

Center-based allocation

Figure 14: Case of center-based allocation harming future allocations. 2D machine with jobs shown as shaded regions.

Aggarwal [34] ran local search from an intial greedy assignment. Hoefler and Snir [31] use a search based on simulated annealing that tests whether random swaps improve an initial solution.

Other task mapping heuristics have also been tried with non-contiguous allocations, including greed [35], genetic algorithms [36], simulated annealing [37], and spectral methods [38].

\section{Discussion}

We have shown that the local search strategy GSEARCH can improve the running time of stencil-based applications while meaningfully reducing the variation between runs. We also showed a significant interaction between the allocation and mapping algorithms. GSEARCH modestly improves mappings based on the linear allocation algorithm and needs relatively fewer swaps to do so, but gives more improvement at higher cost with MC1x1. In practical terms, GSEARCH's benefit seems proportional to the number of swaps performed.

The reason that center-based allocations were subject to greater improvement is unclear, but we speculate that the linear algorithm does a better job maintaining large contiguous groups of free nodes; it gives allocations as wide as the machine rather than allocating cubes and potentially leaving nodes that are far from other free nodes, thus harming future allocations; see Figure 14. The situation can progressively worsen with subsequent allocations. A similar explanation has been given for allocation algorithms that excessively optimize individual allocations [15].

Another question raised by our work is the apparent anomaly of GЕом improving between the $2 \mathrm{~K}$ and $4 \mathrm{~K}$ sizes. Leung et al. [7] reported a similar phenomenon in a slightly different setting. Specifically, they reported a slight decline in GEOM running time for miniGhost going from $2 \mathrm{~K}$ nodes to $4 \mathrm{~K}$ nodes when each MPI rank ran on 4 cores rather than 16 as in our experiments. One explanation is that this improvement comes from the job using such a large part of the machine; this necessarily means that many allocated nodes are contiguous, simplifying task mapping and 
reducing interference from other jobs since there are fewer of them. This explanation is not entirely consistent with Figures 3 and 4, however, since they show GSEARCH reducing average hops for all job sizes. Possible reasons for this discrepancy include an effect from machine or job shapes, areas where the simulation makes simplifications. It is also possible that average hops and job running time diverge in this case, despite the correlation between them and other support for the use of average hops as a proxy for running time (like the benefits from GSEARCH).

Beyond these questions, a logical next step in this work is to develop a fully parallel implemenation of GSEARCH; our current version computes the mapping serially even though all nodes in the allocation are available. The search space of possible swaps is large and most swaps are independent so parallelism should help reduce the search time, but it is not obvious how to decompose the search space or handle swaps that will affect multiple parts of it. One idea is to build on MJ [8], which already investigates different shifts and rotations in parallel.

Another algorithmic idea is to perform the local search to improve theoretical congestion [8], the job's number of overlapping routes, rather than pairwise distance. Theoretical congestion is more highly correlated with running time, but is much harder to compute.

\section{Acknowledgments}

E. Balzuweit, D.P. Bunde, A. Finley, and A.C.S. Lee were partially supported by contract 899808 from Sandia National Laboratories. Sandia National Laboratories is a multi-program laboratory managed and operated by Sandia Corporation, a wholly owned subsidiary of Lockheed Martin Corporation, for the U.S. Department of Energy's National Nuclear Security Administration under contract DE-AC04-94AL85000. We also thank Moe Jette for contributing the LLNL-Atlas trace to the Parallel Workloads Archive.

\section{References}

[1] R. Barrett, C. Vaughan, S. Hammond, D. Roweth, Reducing the bulk in the bulk synchronous parallel mode, Parallel processing letters.

[2] A. Bhatele, L. Kale, Benefits of topology-aware mapping for mesh topologies, Parallel Processing Letters 18 (4) (2008) 549-566.

[3] F. Gygi, E. W. Draeger, M. Schulz, B. de Supinski, J. Gunnels, V. Austel, J. Sexton, F. Franchetti, S. Kral, C. Ueberhuber, J. Lorenz, Large-scale electronic structure calculations of high-Z metals on the BlueGene/L platform, in: Proc. ACM/IEEE Conf. High Performance Networking and Computing (SC), 2006.

[4] H. Subramoni, S. Potluri, K. Kandalla, B. Barth, J. Vienne, J. Keasler, K. Tomko, K. Schulz, A. Moody, D. K. Panda, Design of a scalable infiniband topology service to enable network-topology-aware placement of processes, in: Proc. Conf. High Performance Computing, Networking, Storage and Analysis (SC), 2012.

[5] S. von Alfthan, I. Honkonen, M. Palmroth, Topology aware process mapping, in: Applied Parallel and Scientific Computing, Vol. 7782 of LNCS, Springer, 2013, pp. 297-308.

[6] A. Bhatele, K. Mohror, S. Langer, K. Isaacs, There goes the neighborhood: Performance degradation due to nearby jobs, in: Proc. Conf. High Performance Computing, Networking, Storage and Analysis (SC), 2013, p. 41.

[7] V. Leung, D. Bunde, J. Ebbers, S. Feer, N. Price, Z. Rhodes, M. Swank, Task mapping stencil computations for non-contiguous allocations, in: Proc. 19th ACM SIGPLAN Symp. Principles and Practice of Parallel Programming (PPoPP), 2014, pp. 377-378.

[8] M. Deveci, S. Rajamanickam, V. Leung, K. Pedretti, S. Olivier, D. Bunde, Ü. Çatalyürek, K. Devine, Exploiting geometric partitioning in task mapping for parallel computers, in: Proc. 28th IEEE Intern. Parallel and Distributed Processing Symp. (IPDPS), 2014.

[9] V. Leung, E. Arkin, M. Bender, D. Bunde, J. Johnston, A. Lal, J. Mitchell, C. Phillips, S. Seiden, Processor allocation on Cplant: Achieving general processor locality using one-dimensional allocation strategies, in: Proc. 4th IEEE Intern. Conf. on Cluster Computing, 2002, pp. 296-304.

[10] A. Bhatelé, G. Gupta, L. Kalé, I.-H. Chung, Automated mapping of regular communication graphs on mesh interconnects, in: Proc. Intern. Conf. High Performance Computing (HiPC), 2010. 
[11] E. Balzuweit, D. Bunde, V. Leung, A. Finley, A. Lee, Local search to improve task mapping, in: Proc. 7th Intern. Workshop Parallel Programming Models and Systems Software for High-End Computing (P2S2), 2014.

[12] V. Lo, K. Windisch, W. Liu, B. Nitzberg, Non-contiguous processor allocation algorithms for mesh-connected multicomputers, IEEE Trans. Parallel and Distributed Systems 8 (7) (1997) 712-726.

[13] P. Walker, D. Bunde, V. Leung, Faster high-quality processor allocation, in: Proc. 11th LCI Intern. Conf. HighPerformance Clustered Computing, 2010.

[14] C. Albing, N. Troullier, S. Whalen, R. Olson, J. Glenski, H. Mills, Topology, bandwidth and performance: A new approach in linear orderings for application placement in a 3D torus, in: Proc. Cray User Group, 2011.

[15] M. Bender, D. Bunde, E. Demaine, S. Fekete, V. Leung, H. Meijer, C. Phillips, Communication-aware processor allocation for supercomputers: Finding point sets of small average distance, Algorithmica 50 (2) (2008) 279-298.

[16] J. Mache, V. Lo, K. Windisch, Minimizing message-passing contention in fragmentation-free processor allocation, in: Proc. 10th IASTED Intern. Conf. Parallel and Distributed Computing and Systems, 1997, pp. 120-124.

[17] S. Krumke, M. Marathe, H. Noltemeier, V. Radhakrishnan, S. Ravi, D. Rosenkrantz, Compact location problems, Theoretical Computer Science 181 (2) (1997) 379-404.

[18] F. Kaplan, J. Meng, A. Coskun, Optimizing communication and cooling costs in HPC data centers via intelligent job allocation, in: Proc. Intern. Green Computing Conference (IGCC), 2013, pp. 1-10.

[19] J. Ang, D. Doerfler, S. Dosanjh, S. Hemmert, K. Koch, J. Morrison, M. Vigil, The Alliance for Computing at the Extreme Scale, in: Proc. 52nd Cray User Group, 2010.

[20] Los Alamos National Laboratory, High-performance computing: Cielo supercomputer, http://www. lanl.gov/ orgs/hps/cielo/index.html (viewed September 2013).

[21] Top 500 Supercomputer Sites, http://www.top500.org/.

[22] R. Barrett, C. Vaughan, M. Heroux, MiniGhost: A miniApp for exploring boundary exchange strategies using stencil computations in scientific parallel computing, Tech. Rep. SAND2011-5294832, Sandia National Laboratories (2011).

[23] E. Hertel, R. Bell, M. Elrick, A. Farnsworth, G. Kerley, J. McGlaun, S. Petney, S. Silling, P. Taylor, L. Yarrington, CTH: A software family for multi-dimensional shock physics analysis, in: Proc. 19th International Symposium on Shock Waves, 1993.

[24] R. Barrett, S. Hammond, C. Vaughan, D. Doerfler, J. Luitjens, D. Roweth, Navigating an evolutionary fast path to exascale, in: Proc. 3rd Intern. Workshop Performance Modeling, Benchmarking and Simulation of High Performance Computing Systems (PMBS), 2012.

[25] D. Bunde, V. Leung, PReMAS: Simulator for resource management, in: Proc. 10th Intern. Workshop Scheduling and Resource Management for Parallel and Distributed Systems (SRMPDS), 2014.

[26] D. Feitelson, The parallel workloads archive, http://www.cs.huji.ac.il/labs/parallel/workload/ index.html.

[27] Y. Aridor, T. Domany, O. Goldshmidt, J. Moreira, E. Shmueli, Resource allocation and utilization in the Blue Gene/L supercomputer, IBM J. Research and Development 49 (2/3) (2005) 425.

[28] V. Subramani, R. Kettimuthu, S. Srinivasan, J. Johnson, P. Sadayappan, Selective buddy allocation for scheduling parallel jobs on clusters, in: Proc. 4th IEEE Intern. Conf. on Cluster Computing, 2002, pp. 107-116.

[29] H. Yu, I.-H. Chung, J. Moreira, Topology mapping for Blue Gene/L supercomputer, in: Proc. ACM/IEEE Conf. High Performance Networking and Computing (SC), 2006.

[30] M. Heroux, D. Doerfler, P. Crozier, J. Willenbring, H. Edwards, A. Williams, M. Rajan, E. Keiter, H. Thornquist, R. W. Numrich, Improving performance via mini-applications, Tech. Rep. SAND2009-5574, Sandia National Laboratories (2009).

[31] T. Hoefler, M. Snir, Generic topology mapping strategies for large-scale parallel architectures, in: Proc. 25rd ACM Intern. Conf. Supercomputing (ICS), 2011.

[32] K. Schloegel, G. Karypis, V. Kumar, Parallel static and dynamic multi-constraint graph partitioning, Concurrency and Computation: Practice and Experience 14 (3) (2002) 219-240.

[33] S. Bokhari, On the mapping problem, IEEE Trans Computers C-30 (3).

[34] S.-Y. Lee, J. K. Aggarwal, A mapping strategy for parallel processing, IEEE Trans. Computers C-36 (4).

[35] T. Agarwal, A. Sharma, L. Kalé, Topology-aware task mapping for reducing communication contention on large parallel machines, in: Proc. 20th IEEE Intern. Parallel and Distributed Processing Symp. (IPDPS), 2006.

[36] T. Chockalingam, S. Arunkumar, Genetic algorithm based heuristics for the mapping problem, Computers and Operations Research 22 (1) (1995) 55-64.

[37] S. Bollinger, S. Midkiff, Heuristic technique for processor and link assignment in multicomputers, IEEE Trans. Computers 40 (3).

[38] I.-H. Chung, C.-R. Lee, J. Zhou, Y.-C. Chung, Hierarchical mapping for HPC applications, in: Proc. Workshop on Large-Scale Parallel Processing, 2011, pp. 1810-1818. 